\title{
ON THE VALUE DISTRIBUTION OF ITERATED ENTIRE FUNCTIONS
}

\author{
ZHENG JIAN-HUA
}

(Received 2 March 1998; revised 24 August 1998)

Communicated by P. C. Fenton

\begin{abstract}
Let $f$ be a transcendental entire function and denote the $n$-th iterate of $f$ by $f_{n}$. For $n \geq 2$, we give an explicit estimate of the number of periodic points of $f$ with period $n$, that is, fix-points of $f_{n}$ which are not fix-points of $f_{k}$ for $1 \leq k<n$.
\end{abstract}

1991 Mathematics subject classification (Amer. Math. Soc.): primary 30D05, 58F20.

Keywords and phrases: entire function, iteration, periodic points.

\section{Introduction and results}

We begin by introducing the following fundamental notation and definitions. Let $f(z)$ be a transcendental entire function. We denote by $f_{n}(z)$ the $n$-th iterate of $f(z)$ which is defined by $f_{0}(z)=z, f_{1}(z)=f(z), f_{n}(z)=f\left(f_{n-1}(z)\right)=f_{n-1}(f(z))$. A point $z_{0}$ is said to be a periodic point with period $n$ if $f_{n}\left(z_{0}\right)=z_{0}$ but for $0<k<n$, $f_{k}\left(z_{0}\right) \neq z_{0}$. And according as the modulus of its multiplier, $\lambda=f_{n}^{\prime}\left(z_{0}\right)$, satisfies $|\lambda|<1,|\lambda|=1$, or $|\lambda|>1$, we classify the periodic point $z_{0}$ of period $n$ into, respectively, attracting, indifferent or repelling. We denote by $\rho(f)$ the order of $f(z)$; by $F$ a set on the positive real axis with finite logarithmic measure, not necessarily the same at each occurrence; and by $v(r, f)$ the central index of the power series of $f$ expanded at $z=0$. We shall use the standard notation of Nevanlinna theory, such as $T(r, f), \bar{N}(r, f)$ and $N(r, f)$ (see [7]).

The following is the main result of this paper.

This work is supported by NSF of China.

(C) 1999 Australian Mathematical Society 0263-6115/99 $\$ A 2.00+0.00$ 
THEOREM 1. Let $f(z)$ be a transcendental entire function. Then for $n \geq 2$, there exists an unbounded sequence of $r$ such that either

$$
(1-o(1)) \frac{1}{1403} \log M\left(r, f_{n}\right)<\overline{N_{n}}\left((e+1) r, \frac{1}{f_{n}-z}\right),
$$

or for every finite complex number a,

$$
(1-o(1)) n\left(r^{d}, \frac{1}{f_{n}-a}\right)<\overline{n_{n}}\left(r, \frac{1}{f_{n}-z}\right)
$$

where $\overline{N_{n}}\left(r, 1 /\left(f_{n}-z\right)\right)$ is the counting function corresponding to the number $\overline{n_{n}}\left(r, 1 /\left(f_{n}-z\right)\right)$ of periodic points of $f$ with period $n$, ignoring multiplicities, in $|z| \leq r$, and $d \geq(1 / 1500)^{2}$.

We remark that Theorem 1 gives an estimate of the number of periodic points of period $n$ of $f$ and confirms the conjecture, posed by Baker ([8, Problem 2.20]) and proved by Bergweiler [5], that for $n \geq 2$, there exist infinitely many periodic points of period $n$. For references of the background of this subject we refer the reader to [2], [3] and [5].

The method used in this paper, and which is in essence due to [5] and [11], enables us to prove the following theorem.

THEOREM 2. Let $f(z)$ be a transcendental entire function and $P(z)$ a non-constant polynomial. Then for $n \geq 2$, there exists an unbounded sequence of $r$ such that

$$
(1-o(1)) \frac{1}{1403} \log M\left(r^{d}, f_{n}\right)<N\left(r, \frac{1}{f_{n}-P}\right),
$$

where $d \geq 1 / 1500$.

We remark that under the assumption of $\rho(f)<1 / 2$, Baker [2] showed that the inequality

$$
\log M\left(r^{d}, f_{n}\right)<N\left(r, \frac{1}{f_{n}-z}\right)+O(\log r)
$$

holds for all sufficiently large $r$, where $d$ depends on $n$ and $\rho(f)$, and $d \rightarrow 0$, as $n \rightarrow \infty$.

Finally, from the proof of the above theorems given below, we immediately deduce the following theorem. 
THEOREM 3. Let $f(z)$ and $g(z)$ be two transcendental entire functions and $P(z)$ a non-constant polynomial. Assume that $f(z)$ has a finite deficient value or a finite asymptotic value. Then we have

$$
\begin{aligned}
\frac{1}{1083} \log M(r, f(g)) & <\bar{n}\left(\chi, \frac{1}{f(g)-P}\right)+O(\log r v(r, g)) \\
& \leq \bar{N}\left(e \chi, \frac{1}{f(g)-P}\right)+O(\log r v(r, g)), r \notin F,
\end{aligned}
$$

where $\chi=r+c r / v(r, g), c=20(4+\pi)$.

Note that each of the conditions which $f(z)$ satisfies in Theorem 3 implies $\rho(f) \geq$ $1 / 2$.

THEOREM 4. Let $f(z), g(z)$ and $P(z)$ be given as in Theorem 3. Assume that $\rho(f)<1 / 2$. Then there is a constant $c>1$ such that for all sufficiently large $r$, we have

$$
(1-o(1)) \frac{1}{d} T\left(r^{d}, f(g)\right)<N\left(r, \frac{1}{f(g)-P}\right)
$$

where $d=\min \{1 / 1085, c /(\cos \pi \rho-\varepsilon)\}$, in which $\varepsilon>0$ is chosen sufficiently small so that $\cos \pi \rho-\varepsilon>0$.

\section{Some results needed in proofs}

First of all, let us establish a different form of Nevanlinna's second fundamental theorem. It is well-known that the second fundamental theorem of Nevanlinna can be re-expressed as follows:

Let $F(z)$ be meromorphic in $|z| \leq R$. If $F(0) \neq 0,1, \infty$ and $F^{\prime}(0) \neq 0$, then for $0<r<R$, we have

$$
\begin{aligned}
T(r, F)< & \bar{N}(r, F)+\bar{N}\left(r, \frac{1}{F}\right)+\bar{N}\left(r, \frac{1}{F-1}\right)+m\left(r, \frac{F^{\prime}}{F}\right) \\
& +m\left(r, \frac{F^{\prime}}{F-1}\right)+\log \left|\frac{F(0)(F(0)-1)}{F^{\prime}(0)}\right|+\log 2 .
\end{aligned}
$$

From (5) and by the same argument as in Yang [10, p. 64], we deduce the following lemma. 
LEMMA 1. Let $F(z)$ be meromorphic in $|z| \leq R(<\infty)$. If $F(0) \neq 0,1, \infty$ and $F^{\prime}(0) \neq 0$, then for $0<r<R$,

$$
\begin{aligned}
T(r, F)< & 2\left\{\bar{N}(R, F)+\bar{N}\left(R, \frac{1}{F}\right)+\bar{N}\left(R, \frac{1}{F-1}\right)\right\}+191 \\
& +4 \log ^{+}|F(0)|+2 \log ^{+} \frac{1}{R\left|F^{\prime}(0)\right|}+12 \log ^{+} \frac{R}{R-r} .
\end{aligned}
$$

We further deduce the following consequence of Lemma 1.

LEMMA 2. Let $F(z)$ be holomorphic in $|z| \leq R$ and let

$$
N:=\bar{n}\left(R, \frac{1}{F}\right)+\bar{n}\left(R, \frac{1}{F-1}\right),
$$

where $\vec{n}(R, *)$ denotes the number of distinct poles of $*$ in $|z|<R$. Then for $0<r<R$, we have

$$
\log M(r, F)<\frac{20 R}{R-r}\left(2 N \log \frac{80 e R}{R-r}+195+4 \log ^{+}\left|F\left(z_{0}\right)\right|+12 \log ^{+} \frac{5 R}{R-r}\right),
$$

for all $z_{0}$ in $|z|<(R-r) / 5$, except possibly for the points in the union $(\gamma)$ of certain disks, the total sum of whose radii does not exceed $(R-r) / 20$.

PROOF. Let $a_{v}(v=1, \ldots, N)$ be all the distinct zeros and distinct 1-points of $F(z)$ in $|z|<R$. By the Boutroux-Cartan theorem, we have

$$
\prod_{v=1}^{N}\left|z-a_{v}\right|>\mu^{N}, \quad \mu=\frac{R-r}{40 e},
$$

except for the points in the union $(\gamma)$ of certain disks, the total sum of whose radii is at most $2 e \mu<(R-r) / 20$.

Let $z_{0} \notin(\gamma)$ be a point in $|z|<(R-r) / 5$, and in the annulus $I:=\left\{r+\frac{2}{5}(R-r) \leq\right.$ $\left.\left|z-z_{0}\right| \leq r+\frac{3}{5}(R-r)\right\}$, we can find a circle $\left|z-z_{0}\right|=t$ which does not intersect with $(\gamma)$. We can do this because the distance between the inner and outer circles of $I$ is $(R-r) / 5>4 e \mu$. Set

$$
|F(w)|=\max _{\left|z-z_{0}\right|=t}|F(z)|
$$

and draw a segment $\overline{z_{0} w}$ connecting $z_{0}$ and $w$. Then we can construct a curve $L$ from $\overline{z_{0} w}$ by replacing the part of $\overline{z_{0} w}$ in the interior of $(\gamma)$ by the corresponding boundary arcs of the discs. Obviously, the length of $L$ does not exceed

$$
r+\frac{3}{5}(R-r)+\pi \frac{R-r}{10}<R \text {. }
$$


We need to consider two cases.

Case(a). The inequality $\left|F^{\prime}(z)\right|<1 / R$ holds uniformly on $L$. Obviously,

$$
|F(w)| \leq\left|F\left(z_{0}\right)\right|+\left|\int_{L} F^{\prime}(z) d z\right| .
$$

Since $\{z:|z|<r\} \subset\left\{z:\left|z-z_{0}\right|<t\right\}$, we have

$$
\log M(r, F) \leq \log M\left(t, z_{0}, F\right) \leq \log ^{+}\left|F\left(z_{0}\right)\right|+\log 2 .
$$

Case(b). There exists a point $z_{1}$ on $L$ such that $\left|F^{\prime}\left(z_{1}\right)\right|=1 / R$ and $\left|F^{\prime}(z)\right|<1 / R$ holds uniformly on $L$ from $z_{0}$ to $z_{1}$. If $\left|F^{\prime}\left(z_{0}\right)\right| \geq 1 / R$, we define $z_{1}=z_{0}$. Then

$$
\left|F\left(z_{1}\right)\right| \leq\left|F\left(z_{0}\right)\right|+1 .
$$

Set $\gamma_{0}=\left|w-z_{1}\right|+(R-r) / 10, \gamma_{1}=\left|w-z_{1}\right|+(R-r) / 5$ and $\gamma_{2}=\left|w-z_{1}\right|+2(R-r) / 5$. It is easy to see that $\left\{z:\left|z-z_{1}\right| \leq \gamma_{2}\right\} \subset\{z:|z|<R\}$. Let $a_{v}\left(v=1,2, \ldots, N_{1}\right)$ be all the distinct zeros and distinct 1-points of $F(z)$ in $\left|z-z_{1}\right| \leq \gamma_{2}$. Since $z_{1} \notin(\gamma)$, from (6) it follows that

$$
(2 R)^{N-N_{1}} \prod_{v=1}^{N_{1}}\left|z_{1}-a_{v}\right| \geq\left(\prod_{v=1}^{N_{1}}\left|z_{1}-a_{v}\right|\right)\left(\prod_{v=N_{1}+1}^{N}\left|z_{1}-a_{v}\right|\right)>\mu^{N},
$$

and further

$$
\left(\frac{80 e R}{R-r}\right)^{N} \geq \prod_{v=1}^{N_{1}} \frac{R}{\left|z_{1}-a_{v}\right|}
$$

Thus we have

$$
\bar{N}\left(\gamma_{2}, z_{1}, \frac{1}{F}\right)+\bar{N}\left(\gamma_{2}, z_{1}, \frac{1}{F-1}\right) \leq N \log \frac{80 e R}{R-r} .
$$

From (7), and using Lemma 1 in the disk $\left\{\left|z-z_{1}\right|<\gamma_{2}\right\}$, it follows that

$$
\begin{aligned}
\log M(r, F) & \leq \log |F(w)| \leq \log M\left(\gamma_{0}, z_{1}, F\right) \\
& \leq \frac{\gamma_{1}+\gamma_{0}}{\gamma_{1}-\gamma_{0}} T\left(\gamma_{1}, z_{1}, F\right) \\
& \leq \frac{20 R}{R-r}\left(2 N \log \frac{80 e R}{R-r}+191+4 \log ^{+}\left|F\left(z_{1}\right)\right|+12 \log ^{+} \frac{\gamma_{2}}{\gamma_{2}-\gamma_{1}}\right) \\
& \leq \frac{20 R}{R-r}\left(2 N \log \frac{80 e R}{R-r}+195+4 \log ^{+}\left|F\left(z_{0}\right)\right|+12 \log ^{+} \frac{5 R}{R-r}\right) .
\end{aligned}
$$

Thus Lemma 2 follows. 
LEMMA 3. Let $g(z)$ be a transcendental entire function. Assume that $c>0, K>0$ and $\eta>0$. Suppose that $\left|z_{0}\right|=r \notin F,\left|g\left(z_{0}\right)\right| \geq \eta M(r, g)$ and $|\sigma|<K$. Then there exists a unique such that $|v(r, g) s-\sigma|=o(1)$ and

$$
g\left(z_{0} e^{s}\right)=g\left(z_{0}\right) e^{\sigma},
$$

and a function $\tau(z)$ defined and analytic on $\left|z-z_{0}\right| \leq c r / v(r, g)$, and satisfying

$$
|\tau(z) v(r, g)-2 \pi i|=o(1)
$$

such that

$$
g\left(z e^{\tau(z)}\right)=g(z) .
$$

Lemma 3 is in essence proved by using Wiman-Valiron theory [9] and was explicitly developed by Bergweiler [4]. The following lemma is due to Clunie [6].

LEMMA 4. Let $f(z)$ and $g(z)$ be two transcendental entire functions. Then

$$
M(r, f(g))=M((1-o(1)) M(r, g), f), r \notin F .
$$

The following lemma is due to Baker [1]. It is often used in the proof of the main theorem of Bergweiler [5], as well as in this paper.

LEMMA 5. Let $f(z)$ be an entire function. For any $B>A$, if $|f(z)|<R$ in $|z|<A$, but $|f(z)|>R$ on $|z|=B$, then there exists a simple curve $\Gamma$ in $\{A \leq|z|<B\}$ going around the origin once such that $|f(z)|=R$ on $\Gamma$

By analyzing the proof of [2, Theorem 1], we are immediately able to prove the following lemma.

LEMMA 6. Let $F(z)$ be a transcendental entire function and $P(z)$ be a polynomial with the first term $a_{m} z^{m}\left(a_{m} \neq 0\right)$. Assume that, for some $\sigma>1$ and some $r$ satisfying

$$
2\left|a_{m}\right| r^{m \sigma} \geq|P(z)|, \quad \text { on }|z|=r^{\sigma},
$$

there exists a simple closed curve $\Gamma \subset\left\{r<|z|<r^{\sigma}\right\}$ going around the origin once, on which

$$
|F(z)| \geq M>2\left|a_{m}\right| r^{m \sigma} .
$$

Then

$$
N\left(r^{\sigma}, \frac{1}{f-P}\right)>\log \left(M-2\left|a_{m}\right| r^{m \sigma}\right)-O(\log r)
$$




\section{Proofs of theorems}

We begin with the proof of Theorem 2. Put $g=f_{n-1}$, so that $f(g)=f_{n}$. Assume that for sufficiently large $r \notin F$, where $F$ is the set arising from Lemmas 3 and 4 , we have

$$
\bar{n}\left(\chi, \frac{1}{f(g)-P}\right) \leq \frac{1}{1403} \log M(r, f(g)),
$$

where $\chi=r+2 R, R=20(4+\pi) r / v(r, g)$.

We want to prove the following

Claim. In

$$
e^{-3} M(r, g) \leq|w| \leq e^{3} M(r, g),
$$

there exists a circle $\Gamma_{0}:|w|=\xi_{r}$ on which we have

$$
(1-o(1)) \frac{1}{1403} \log M(r, f(g)) \leq \log |f(w)| .
$$

The same inequality still holds with $f$ and $g$ interchanged.

Now we choose a point $z_{0}$ on $|z|=r$ such that

$$
\left|f\left(g\left(z_{0}\right)\right)\right|=M(r, f(g))=M((1-o(1)) M(r, g), f),
$$

then $M(r, g) \geq\left|g\left(z_{0}\right)\right| \geq(1-o(1)) M(r, g)$. Application of Lemma 3 to $z_{0}$ and $g$ implies the existence of an analytic function $\tau(z)$ defined in $\left|z-z_{0}\right| \leq R$, where $R=20(4+\pi) r / v(r, g)$, such that

$$
|\tau(z) v(r, g)-2 \pi i|<1
$$

and

$$
g\left(z e^{\tau(z)}\right)=g(z)
$$

Set $k(z)=z e^{\tau(z)}$ and

$$
h(z)=\frac{f(g(z))-P(z)}{P(k(z))-P(z)},
$$

in $\left|z-z_{0}\right| \leq R$. It is easy to prove that $h(z)$ is analytic in $\left|z-z_{0}\right| \leq R$. Let $(\gamma)$ be the union of exceptional disks, the total sum of whose radii does not exceed $R / 80$, 
the existence of which follows from Lemma 2, taking $F=h, r=\frac{3}{4} R$ and the disk $\left|z-z_{0}\right|<R$.

For $w$ satisfying (9) we can write $w=e^{\sigma} g\left(z_{0}\right)$, where $|\operatorname{Re} \sigma| \leq 3,|\operatorname{Im} \sigma| \leq \pi$. From Lemma 3, we can find a unique $s$ such that $v(r, g) s=\sigma+o(1)$ and $g\left(z_{0} e^{s}\right)=$ $g\left(z_{0}\right) e^{\sigma}=w$. Put $u=z_{0} e^{s}$, then

$$
\left|u-z_{0}\right|=r\left|e^{s}-1\right| \leq \frac{(4+\pi) r}{v(r, g)}=\frac{R}{20} .
$$

It is obvious that the mapping $w=e^{\sigma} g\left(z_{0}\right)$ maps the segment

$$
L_{1}=\left\{w: \arg w=\theta \text { and } e^{-3} M(r, g) \leq|w| \leq e^{3} M(r, g)\right\}
$$

into a segment which contains the segment

$$
L_{2}=\left\{\sigma: \arg g\left(z_{0}\right)+\operatorname{Im} \sigma=\theta \text { and }-\frac{8}{3} \leq \operatorname{Re} \sigma \leq \frac{8}{3}\right\},
$$

and that $L_{2}$ is mapped by $u=z_{0} e^{s}, v(r, g) s=\sigma+o(1)$, into a curve $L_{3}$, the diameter of which is at least $R / 30$. And therefore we can find a circle $\Gamma_{0}:|w|=\xi_{\text {r }}$ in $\left\{e^{-3} M(r, g) \leq|w| \leq e^{3} M(r, g)\right\}$ which is such that $u$ corresponding to $w$ on $\Gamma_{0}$ is not contained in $(\gamma)$.

Obviously, by (11), a simple calculation implies that

$$
\left\{z:\left|z-z_{0}\right|<R\right\} \subset\{z:|z|<r+R\}
$$

and

$$
\left\{k(z):\left|z-z_{0}\right|<R\right\} \subset\{z:|z|<r+2 R=\chi\} .
$$

Thus it follows from (12) and (13) that

$$
\bar{n}\left(R, z_{0}, h\right)+\bar{n}\left(R, z_{0}, \frac{1}{h}\right)+\bar{n}\left(R, z_{0}, \frac{1}{h-1}\right) \leq \bar{n}\left(\chi, \frac{1}{f(g)-P}\right) .
$$

By applying Lemma 2 to $h(z)$ in $\left|z-z_{0}\right|<R$, for $u \notin(\gamma)$ satisfying $\left|u-z_{0}\right|<$ $\left(R-\frac{3}{4} R\right) / 5=R / 20$, we have

$$
\begin{aligned}
\log \left|h\left(z_{0}\right)\right| \leq & \log M\left(\frac{3}{4} R, z_{0}, h\right) \\
< & \frac{20 R}{R-\frac{3}{4} R}\left\{2 \bar{n}\left(\chi, \frac{1}{f(g)-P}\right) \log \frac{80 e R}{R-\frac{3}{4} R}+195+4 \log ^{+}|h(u)|\right. \\
& \left.\quad+12 \log ^{+} \frac{5 R}{R-\frac{3}{4} R}\right\} \\
< & 1083 \bar{n}\left(x, \frac{1}{f(g)-P}\right)+O(1)+320 \log ^{+}|h(u)| .
\end{aligned}
$$


On the other hand, from (13) it follows that

$$
\log \left|h\left(z_{0}\right)\right|>\log M(r, f(g))-O(\log r v(r, g)) .
$$

Hence, by (13) we have

$$
\begin{aligned}
\log M(r, f(g)) & \leq 1083 \bar{n}\left(\chi, \frac{1}{f(g)-P}\right)+O(\log r v(r, g))+320 \log ^{+}|h(u)| \\
& \leq 1083 \bar{n}\left(x, \frac{1}{f(g)-P}\right)+O(\log r v(r, g))+320 \log ^{+}|f(w)|,
\end{aligned}
$$

and further from (8)

$$
\frac{1}{1403} \log M(r, f(g)) \leq \log ^{+}|f(w)|+O(\log r v(r, g)),
$$

on $\Gamma_{0}$. Thus the claim is proved.

Now choose a $t \notin F$ in the interval $\left((M(r, f) / 2)^{1 / 1425},(M(r, f) / 2)^{q}\right)$, where $q=\frac{1}{2}(1 / 1403+1 / 1425)$. Then from the claim, with alternation of $f$ and $g$, and the fact that $\log M(r, g)$ is convex with respect to $\log r$, we have on $\Gamma_{0}:|w|=\xi_{r} \subset$ $\left\{e^{-3} M(r, f) \leq|w| \leq e^{3} M(r, f)\right\}$

$$
\begin{aligned}
\log |g(w)| & \geq(1-o(1)) \frac{1}{1403} \log M(r, g(f)) \\
& \geq(1-o(1)) \frac{1}{1403} \log M(M(r, f) / 2, g) \\
& >\log M(t, g)+3,
\end{aligned}
$$

so that

$$
|g(w)|>e^{3} M(t, g) \geq \xi_{t} .
$$

On the other hand, obviously for $|w|<t^{\alpha}, \alpha=29 / 30$, we have

$$
|g(w)|<e^{-3} M(t, g) \leq \xi_{t} .
$$

Then there exists a simple curve $\Gamma \subset\left\{t^{\alpha}<|w|<\xi_{r}\right\} \subset\left\{t^{\alpha} \leq|w|<t^{1450}\right\}=\{\tilde{t} \leq$ $\left.|w|<\tilde{t}^{1500}\right\}, \tilde{t}=t^{\alpha}$, which goes around the origin once and on which $|g(w)|=\xi_{t}$. Applying the claim once more implies that

$$
\log ^{+}|f(g(w))| \geq(1-o(1)) \frac{1}{1403} \log M(t, f(g)) \text {, on } \Gamma .
$$

Then by Lemma 6, we get (2). 
If for an unbounded sequence of $r \notin F$, (8) does not hold, it is easy to deduce (2) from the convexity of $\log M(r, g)$ with respect to $\log r$ and the fact that

$$
\bar{n}\left(\chi, \frac{1}{f(g)-P}\right) \leq \bar{N}\left(e \chi, \frac{1}{f(g)-P}\right) .
$$

Thus the proof of Theorem 2 is complete.

Now we are in position to prove Theorem 3. Conversely, suppose that for $r \notin F$, we have

$$
\bar{n}\left(\chi, \frac{1}{f(g)-P}\right) \leq \frac{1}{1083}(\log M(r, f(g))-O(\log r v(r, g))-320 \log r),
$$

where $O(\log r v(r, g))$ is the quantity occurring in (14). By the same argument as in the proof of the claim, from (14) and (16), we can prove that there exists a circle $\Gamma_{0}:|w|=\xi_{r}$ on which we have

$$
\log r \leq \log |f(w)| .
$$

In fact, first of all, we deduce (14), and by (16) deduce (17). Obviously for any finite number $a$, it follows from (17) that for sufficiently large $r$

$$
\log |f(w)-a|>1, \text { that is, } m\left(\xi_{r}, \frac{1}{f-a}\right)=0,
$$

where $|w|=\xi_{r}$, so $\delta(a, f)=0$ and it is easy to see from (17) that $f$ has no finite asymptotic values, which is a contradiction. Thus (16) does not hold, and Theorem 3 follows.

Before proving Theorem 4 , we need a well-known result on transcendental entire function with order less than $1 / 2$.

LEMMA 7. [1, p. 131, formula (25)]. Let $\varepsilon>0$ be a given number and $h(z)$ an entire function of order $\rho<1 / 2$. Then there exists a constant $c>1$ such that, for all sufficiently large $R$, the interval $\left(R, R^{c}\right)$ contains an $R_{0}$ with

$$
\tilde{m}\left(R_{0}, h\right)>\left\{M\left(R_{0}, h\right)\right\}^{-\varepsilon+\cos \pi \rho},
$$

where $\tilde{m}\left(R_{0}, h\right)$ denotes the minimum modulus of $h(w)$ on $|w|=R_{0}$.

Now we prove Theorem 4. Let $\varepsilon>\varepsilon_{0}>0$ be sufficiently small such that $\cos \pi \rho-\varepsilon>0$. Put $p=(\cos \pi \rho-\varepsilon)^{-1}, p_{0}=\left(\cos \pi \rho-\varepsilon_{0}\right)^{-1}$. By the convexity of $\log M(r, g)$ as a function of $\log r$, we have

$$
M\left(r^{p}, g\right) \geq\left(e^{3} M(r, g)\right)^{p_{0}} .
$$


Application of Lemma 7 to $\varepsilon_{0}$ and $g$ implies the existence of $c>1$ which is such that the interval $\left(r^{p}, r^{c p}\right)$ contains an $R_{0}$ such that on $|z|=R_{0}$,

$$
\begin{aligned}
|g(z)| & \geq \tilde{m}\left(R_{0}, g\right) \geq M\left(R_{0}, g\right)^{1 / p_{0}} \\
& \geq M\left(r^{p}, g\right)^{1 / p_{0}} \geq e^{3} M(r, g) \\
& >\xi_{r} .
\end{aligned}
$$

On the other hand, it is obvious that $|g(z)|<M\left(r^{1-\delta}, g\right)<e^{-3} M(r, g) \leq \xi_{r}$ in $|z|<r^{1-\delta}$, where $\delta$ is a sufficiently small positive number. Then it follows from Lemma 5 that there exists a curve $\Gamma \subset\left\{r^{1-\delta} \leq|z|<r^{c p}\right\}$ which contains the origin in its interior and on which $|g(z)|=\xi_{r}$.

If for $r \notin F$, (16) with $320 c p(\operatorname{deg} P+1) \log r$ instead of $320 \log r$ does not hold, then (4) immediately follows. Now we assume such (16) holds, then we have (17) with $c p(\operatorname{deg} P+1) \log r$ instead of $\log r$ on $|w|=\xi_{r}$, that is, $\log ^{+}|f(w)| \geq$ $c p(\operatorname{deg} P+1) \log r,|w|=\xi_{r}$. Then on $\Gamma$ we have

$$
\log ^{+}|f(g(z))| \geq c p(\operatorname{deg} P+1) \log r>\log (|P(z)|+1+a)
$$

where $a$ is a complex number such that $N(r, 1 /(f(g)-a))=(1-o(1)) T(r, f(g))$. By Rouché's theorem, we deduce that $f(g)-P$ has as many zeros as $f(g)-a$ does in the interior of $\Gamma$, and therefore for all sufficiently large $r \geq r_{0}$,

$$
\begin{aligned}
n\left(r^{c p}, \frac{1}{f(g)-P}\right) & \geq n\left(\Gamma, \frac{1}{f(g)-P}\right)=n\left(\Gamma, \frac{1}{f(g)-a}\right) \\
& \geq n\left(r^{1-\delta}, \frac{1}{f(g)-a}\right),
\end{aligned}
$$

where $n(\Gamma, *)$ is the number of poles of $*$ in int $\Gamma$. Further for $r \geq r_{0}$,

$$
\begin{aligned}
N\left(r^{c p}, \frac{1}{f(g)-P}\right) & \geq \frac{c p}{1-\delta} N\left(r^{1-\delta}, \frac{1}{f(g)-a}\right) \\
& =(1-o(1)) \frac{c p}{1-\delta} T\left(r^{1-\delta}, f(g)\right),
\end{aligned}
$$

since for arbitrary positive $s$,

$$
\int_{r_{0}}^{r} \frac{n\left(t^{s}, *\right)}{t} d t=\frac{1}{s} \int_{r_{0}}^{r} \frac{n\left(t^{s}, *\right)}{t^{s}} d t^{s}=\frac{1}{s} N\left(r^{s}, *\right)+O(1) .
$$

By choosing a smaller $\varepsilon$ than the one in the above, we deduce (4). Thus Theorem 4 follows. 
Finally, we prove Theorem 1 . For $0<k<n$ and arbitrarily large $K$, we have

$$
\begin{aligned}
\log M\left(r, f_{n}\right) & \geq \log M\left(\frac{1}{2} M\left(\frac{1}{4} r, f_{n-k}\right), f_{k}\right) \\
& \geq \log M\left(((e+1) r)^{K+1}, f_{k}\right) \\
& \geq(1-o(1)) K T\left((e+1) r, f_{k}\right),
\end{aligned}
$$

and hence

$$
\begin{aligned}
N\left((e+1) r, \frac{1}{f_{k}-z}\right) & \leq T\left((e+1) r, f_{k}\right)+O(\log r) \\
& =o\left(\log M\left(r, f_{n}\right)\right) .
\end{aligned}
$$

On the other hand, it is easy to see that

$$
\overline{N_{n}}\left(r, \frac{1}{f_{n}-z}\right) \geq \bar{N}\left(r, \frac{1}{f_{n}-z}\right)-\sum_{k=1}^{n-1} \bar{N}\left(r, \frac{1}{f_{k}-z}\right) .
$$

Set $g=f_{n-1}$, so that $f(g)=f_{n}$. Therefore, if (8) does not hold for some unbounded sequence of $r \notin F$, then we easily deduce that

$$
\overline{N_{n}}\left((e+1) r, \frac{1}{f_{n}-z}\right) \geq(1-o(1)) \frac{1}{1403} \log M\left(r, f_{n}\right) .
$$

This is (1). Now, we can assume that for all $r \notin F$, we have (8) and further deduce the claim. By the same argument as in the proof of Theorem 2, we can find a simple curve $\Gamma \subset\left\{\tilde{t} \leq|w|<\tilde{t}^{1500}\right\}$ going around the origin once and on which (15) holds. Then the argument of Lemma 5 implies the existence of a simple curve $\Gamma_{0} \subset\left\{\tilde{t}^{1 / 1406} \leq|w|<\tilde{t}^{1500}\right\}$ which surrounds the origin once and on which $|f(g(z))|=M(R, f(g))$, where $R=\tilde{t}^{\beta} \notin F, 1 / 1406 \leq \beta<1 / 1403$. Define $G_{0}=$ int $\Gamma_{0}, G_{1}=g\left(G_{0}\right), G_{2}=f\left(G_{1}\right)$ and $\Gamma_{j}=\partial G_{j}(0 \leq j \leq 2)$. Obviously, by the Maximum Principle, all the $G_{j}$ are simply connected and all the $\Gamma_{j}$ are simple curves and surround the origin once. Assume that $g(z)$ (respectively, $f(z))$ describes $p_{1}$ (respectively, $p_{2}$ ) times the curve $\Gamma_{1}$ (respectively, $\Gamma_{2}$ ) as $z$ describes the $\Gamma_{0}$ (respectively, $\Gamma_{1}$ ) once. Then $f(g)$ has $P_{2}=p_{1} p_{2}$ zeros in $G_{0}$. By Rouché's theorem, $f(g(z))-z$ also has $P_{2}$ zeros in $G_{0}$. From the main ideas in Bergweiler [5], by a little modification of his proof, we can prove that

$$
\overline{P_{2}}=(1-o(1)) P_{2},
$$

where $\overline{P_{2}}$ denotes the number of distinct zeros of $f(g(z))-z$ in $G_{0}$. For completeness, we shall give the proof of (18). First of all, we want to prove that

$$
\overline{f_{j}\left(G_{0}\right)} \subset G_{1}, 0 \leq j<n-1 .
$$


It is obvious that $G_{1}$ is a domain and contains the origin. By the maximum principle, $\overline{f_{j}\left(G_{0}\right)} \subset\left\{|w| \leq M\left(\tilde{t}^{1500}, f_{j}\right)\right\}, 0 \leq j<n-1$. Since on $\Gamma_{0},|f(g(z))|=$ $M(R, f(g)) \geq M\left(\frac{1}{2} M(R, g), f\right)$, we have that $|g(z)| \geq M\left(\tilde{t}^{1500}, f_{j}\right), 0 \leq j<n-1$, on $\Gamma_{0}$, noting that $f$ is transcendental. Thus (19) follows from $\Gamma_{1} \subset g\left(\Gamma_{0}\right) \subset$ $\left\{M\left(\tilde{t}^{1500}, f_{j}\right)<|w|\right\}, 0 \leq j<n-1$.

Let $z_{0} \in G_{0}$ be a zero of $f(g(z))-z$ with multiplicity $m+1$. Then we have that $f_{n}^{\prime}\left(z_{0}\right)=1$ and $z_{0}$ is a periodic point with period $k \leq n$. Let $p$ be the smallest positive integer such that $\left(f_{k}^{\prime}\left(z_{0}\right)\right)^{p}=1$, and further $k p \leq n$, and let $m_{1}+1$ be the multiplicity of the zero $z_{0}$ of $f_{k p}(z)-z$. Then it is easy to see that $s=n /(k p)$ is a positive integer, and $f_{n}=\left(f_{k p}\right)_{s}$, and therefore $m=m_{1}$. By a result of Fatou (see [5, Lemma 6]), it follows that there exist $m / p$ cycles of Leau domains, each of which contains at least one singularity of the inverse function of $f$, and which are in $G_{1}$, by [5, Lemma 8] and (19). Therefore $G_{1}$ contains at least $m / p$ critical points of $f$, for from the claim it is easy to see that $f$ has no asymptotic values. However, $f$ has at most $p_{2}-1$ critical points in $G_{1}$. Hence we have

$$
P_{2}-\widetilde{P_{2}} \leq \sum k m=\sum k p \frac{m}{p} \leq n\left(p_{2}-1\right),
$$

where $\sum$ is taken over all the zeros of $f(g(z))-z$ in $G_{0}$. This implies (18), since $p_{1} \rightarrow \infty$, as $r \rightarrow \infty$.

By $\left(P_{2}\right)_{j}$ we denote the number of zeros of $f_{j}(z)-z$ in $G_{0}$. Obviously, it follows from $\left|f_{n}(z)\right|=M\left(R, f_{n}\right)$ on $\Gamma_{0}$ that $\left|f_{j}(z)\right|>|z|(0 \leq j \leq n-1)$ on $\Gamma_{0}$, and hence $\left(P_{2}\right)_{j}$ is equal to the number of zeros of $f_{j}(z)$ in $G_{0}$, that is, the winding number of $f_{j}\left(\Gamma_{0}\right)$ around the origin. Obviously, $\left(P_{2}\right)_{j} \leq\left(P_{2}\right)_{n-1}=p_{2}$. Since

$$
(1-o(1)) P_{2} \leq \overline{P_{2}} \leq \overline{\left(P_{2}\right)_{n}}+\sum_{j=1}^{n-1}\left(P_{2}\right)_{j} \leq \overline{\left(P_{2}\right)_{n}}+(n-1) p_{2},
$$

we have

$$
\overline{\left(P_{2}\right)_{n}} \geq(1-o(1)) P_{2},
$$

where $\overline{\left(P_{2}\right)_{n}}$ denotes the number of distinct periodic points of period $n$ of $f(z)$ in $G_{0}$, and further we have for arbitrary $a \in G_{0}$

$$
\begin{aligned}
\overline{n_{n}}\left(R^{v}, \frac{1}{f_{n}-z}\right) & \geq \overline{n_{n}}\left(\Gamma_{0}, \frac{1}{f_{n}-z}\right) \geq(1-o(1)) n\left(\Gamma_{0}, \frac{1}{f_{n}-z}\right) \\
& =(1-o(1)) n\left(\Gamma_{0}, \frac{1}{f_{n}-a}\right) \geq(1-o(1)) n\left(R, \frac{1}{f_{n}-a}\right),
\end{aligned}
$$

where $v=1500^{2}$, and $\overline{n_{n}}\left(\Gamma_{0}, 1 /\left(f_{n}-z\right)\right)$ is the number of distinct periodic points of period $n$ of $f$ in int $\Gamma_{0}$.

Thus Theorem 1 follows. 


\section{References}

[1] I. N. Baker, 'Zusammensetzungen ganzer Funktionen', Math. Z. 69 (1958), 121-163.

[2] _ ' 'Fixpoints and iterates of entire functions', Math. $Z .71$ (1959), 146-153.

[3] - 'Some entire functions with fixpoints of every order', J. Austral. Math. Soc. (Series A) 1 (1959/1960), 203-209.

[4] W. Bergweiler, 'On proof of a conjecture of Gross concerning fix-points', Math. Z. 204 (1990), 381-390.

[5] - 'Periodic points of entire functions: Proof of a conjecture of Baker', Complex Variables Theory Appl. 17 (1991), 57-72.

[6] J. Clunie, 'The composition of entire and meromorphic functions', in: Math. essays dedicated to A. J. Macintyre (ed. H. Shankar) (Athens, Ohio, 1970) pp. 75-92.

[7] W. K. Hayman, Meromorphic functions (Clarendon Press, Oxford, 1964).

[8] —.., Research problems in function theory (Athlone Press, London, 1967).

[9] G. Valiron, 'Sur la dérivée des fonctions algébroides', Bull. Soc. Math. France 59 (1929), 17-39.

[10] L. Yang, New researches on value distribution theory, (in Chinese) (Science Press, 1982).

[11] J. H. Zheng and C. C. Yang, 'Further results on fix-points and zeros of entire functions', Trans. Amer. Math. Soc. (1) 347 (1995), 37-50.

\section{Department of Applied Mathematics}

Tsing Hua University

Beijing, 100084

P. R. China

e-mail: jzheng@math.tsinghua.edu.cn 